\title{
A myocardial infarction caused by an epicardial radiofrequency ablation
}

\author{
Authors: Duje Čulina1 ${ }^{1}$, Tomislav Cigić $^{1}$, Vjekoslav Radeljić ${ }^{2}$ \\ 'School of Medicine University of Zagreb, Croatia \\ ${ }^{2}$ University Hospital Centre Sestre Milosrdnice, Clinic for Cardiovascular diseases, Zagreb, Croatia \\ DOI: https://doi.org/10.26800/LV-142-supp5-27
}

\section{Background:}

A myocardial infarction (MI) occurs when blood flow in the coronary blood vessel decreases or stops causing damage to the heart muscle. Cardiac radiofrequency ablation (RFA) is a procedure using catheters inserted through a vein or artery which reach heart and deliver RF energy in the form of heat to modify the heart tissue that triggers or sustains tachycardia or tachyarrhythmia.

\section{Case presentation:}

We present a case of a 66 years old female patient with symptomatic premature ventricular contractions (PVC), which was diagnosed 4 years ago. She was feeling tired and could not tolerate intensified exertion. PVCs manifested as a skipped heartbeat with occasional tachycardia that lasted a few seconds. Three 24-hours Holter-ECG done in the last few months recorded $>20000$ PVCs which is approximately $20 \%$ of daily heartbeats. That is considered a risk factor for tachycardia-induced cardiomyopathy (tachycardiomyopathy). Thus, she was hospitalized for electrophysiology study, during which the PVC focus was located in the left ventricular "summit" area (combining coronary sinus, left main coronary bifurcation). Firstly, RFA with 30 Watts RF energy was unsuccessfully attempted from right ventricle. Same was attempted from left ventricle which achieved partial PVCs elimination. Furthermore, ablation catheter was placed in the coronary sinus and RFA achieved complete PVCs elimination. Soon after the procedure was done, the patient felt chest pain and ECG verified an ST elevation MI (STEMI). Urgent coronary angiography showed an occlusion of the circumflex artery (ACx) ostium, and drug-eluting stent (DES) was implanted. Postprocedural period was uneventful. Therefore, patient experiencing any difficulties, was discharged with recommendation of medications and cardiology follow-up visit.

\section{Conclusion:}

RFA is a minimally invasive procedure that carries a risk of complications, including: $\mathrm{MI}$, bleeding, infection, damage to heart valves or heart's conduction system, pericardial tamponade and venous or arterial thromboembolism.

Keywords: electrophysiology study, DES, PVC, RFA, STEMI 